\title{
A Cellular Bonding AND Adaptive LoAd BALANCING BASED MULTI-SIM GATEWAY FOR MOBILE AD HOC AND SENSOR NETWORKS
}

\author{
Francesco Beritelli, Aurelio La Corte, Corrado Rametta and Francesco Scaglione \\ Dipartimento di Ingegneria Elettrica Elettronica e Informatica, University of Catania, \\ Italy
}

\begin{abstract}
As it is well known, the QoS(quality of service) provided by mobile Internet access point devices is far from the QoS level offered by the common ADSL modem-router due to several reasons: in fact, mobile Internet access networks are not designed to support real-time data traffic because of several drawbacks concerning the wireless medium such as resource sharing, traffic congestion, radio link coverage etc., which impact directly such parameters as delay, jitter, and packet loss rate that are strictly connected to the quality of user experience. The main scope of the present paper is to introduce a dual USIM HSPA gateway for ad hoc and sensors networks thanks to which it will be possible to guarantee a QoS suitable for a series of network-centric application such as real-time communications and monitoring, video surveillance, real-time sensor networks, telemedicine, vehicular and mobile sensor networks and so on. The main idea is to exploit multiple radio access networks in order to enhance the available end-to-end bandwidth and the perceived quality of experience. The scope has been reached by combining multiple radio access with dynamic load balancing and the VPN (virtual private network) bond technique.
\end{abstract}

\section{KEYWORDS}

Mobile Internet Access, Multiple RAN, Cellular Bonding, VPN Bonding, Dynamic Load Balancing, Smart Hot Spotting.

\section{INTRODUCTION}

The rapid and continuous consolidation of Mobile Internet access request together with the significant increase of mobile services provided by third- and fourth generation (3G, HSPA, LTE) networks, have recently created the conditions for a considerable expansion of mobile IP applications and services. Mobile IP networks are not designed to support real-time and/or timecritical traffic because of several drawbacks concerning the wireless medium[1]: resource sharing, traffic congestion, radio link coverage etc., which impact directly such parameters as delay, jitter, and packet losses. These are the main causes of quality degradation of numerous services over the PSTN (public switched telephone network). Some authors of this work proposed the use of multiple SIM (subscriber identity module) devices in order to improve the QoS offered by the cellular radio access technology [2][3][4][5]. Following this paradigm, in the present paper, the authors propose a multiple SIM device, acting as:

1. sink of a wireless sensor network[6], ad hoc network or Internet of Things network[7];

2. gateway between the local wireless domain (ZigBee, Bluetooth, 802.11 ad-hoc mode) and the wide area network domain (radio access domain $3 \mathrm{G}$ or $4 \mathrm{G}$ ). 
The main idea consists in enhancing the connectivity between the local area network (ad hoc, sensors, IoT, etc.) domain and a remote command and control node by exploiting the VPNs bond of two cellular radio access connections coupled with an adaptive load balancing algorithm based on real time evaluation of the available end-to-end bandwidth offered by two different network operators. The authors propose a trivial prototype in order to evaluate the effectiveness of the proposed solution in terms of the enhancement of both the instantaneous available bandwidth and connection availability between the mobile access point and a remote command and control and/or monitoring node. As to the costs/benefits balance, the proposed method on the one hand requires a dual RF module, but on the other hand it is also true that nowadays HSPA or LTE modems have become very cheap and common and there are a lot of free and open source operative systems allowing the implementation of advanced networking functions such as load balancing techniques, VPN creation and bonding, network performance evaluation and so on.

The paper is structured as follows: Section II describes the possible applications and contexts where the proposed approach can offer considerable benefits in terms of reliability and efficiency and gives an overview on the overall system; Section III presents the end-to-end bandwidth measurement algorithm and the adaptive weight assignment procedure; Section IV reports the performance results of a real test bed; finally, in Section $\mathrm{V}$ conclusions are drawn.

\section{SCENARIO AND SYSTEM OVERVIEW}

This paragraph provides a brief overview of both the application scenario and the proposed mechanism based on two key features, the VPN bond between two cellular connections and the adaptive load balancing between the available radio access connections.

\subsection{Application scenario}

Wireless sensor networks (WSNs) and Internet of Things (IoT)have become very commonly used technologies enabling a large number of applications and services in everyday life. Usually the architecture of the above cited networks is based on a certain number of sensors and devices that communicate among them and towards a critical device called sink that has the scope of collecting data from the devices for monitoring, control, statistics, etc. Often the sink is designed to communicate via Internet to a remote command and control (C2) position where a user can operate over the network.

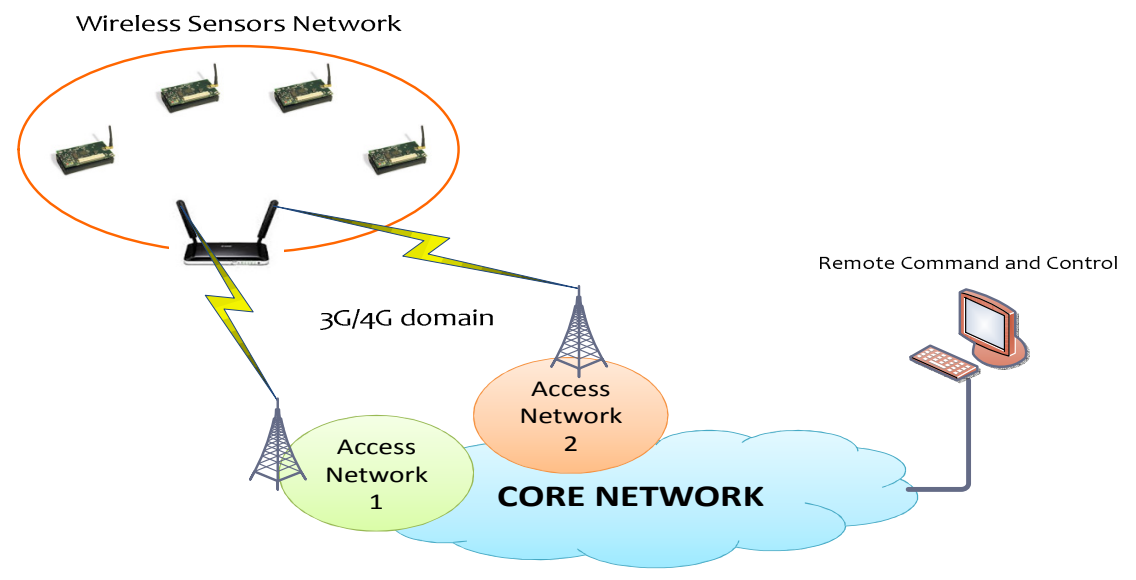

Figure1. Scenario overview. 
International Journal on AdHoc Networking Systems (IJANS) Vol. 5, No. 3, July 2015

With this scope, the sink provides the functionality of gateway with the external IP world. Regarding this aspect, the gateway is equipped with at least two network interfaces: the first is usually a WiFi card operating as access point or a Bluetooth interface acting as master of the ad hoc network or a ZigBee card operating as sink of the sensors network; the second one is a wide area network interface like as wifi-mesh mode, 3G/4G modem, WiMaX or other PMR (private mobile radio) cards in order to communicate with the IP worldaccording to the applicative scenario for which the ad hoc network has been conceived. In particular, when the sensor network or the IoT network are deployed for command and/or control, monitoring, surveillance or similar use in mobile or vehicular contexts and a fixed wide area network connection is not available, it is mandatory to provide a stable, reliable and effective wireless connection towards the remote server in order to guarantee the required $\mathrm{QoS}$ for time-critical and real time applications.

With this aim the authors propose a Dual SIM 3G/4G wireless access point that acts as the sink for the sensors or ad-hoc network and, at the same time, acts as gateway towards the IP core network. To guarantee QoS for time critical and real time applications, the proposed device offers two main features:

1) a VPN bonding between the two radio access connections, thanks to which it is possible to obtain a bandwidth almost equal to the sum of the two available end-to-end bandwidths;

2) a dynamic load balancing algorithm, which is a process that establishes the weights the device gives to the two different radio connections during the movement of the mobile sink/gateway on the base of the instantaneous available end-to-end bandwidth offered by the two different network operators.

It has been observed that in this first phase of the present research the weights are established in order to maximize the cumulative bandwidth but as a future work the authors will implement a more complex algorithm able to dynamically calculate the VPN weights based on the traffic typology (voice, file transfer, best effort, etc) and the related parameters that directly impact on the quality of experience of the end user willing to use the service.

\subsection{System at a glance}

The smart gateway proposed by the authors is based on a well known technique called VPNs bond, usually employed in the Ethernet switch and extended to the cellular domain to counteract some drawbacks connected to the radio access technology. Due to the application of VPNs bond it is possible to balance the data load among the available network interfaces but, for the sake of clarity, it should be highlighted that this technique is much more flexible if compared to the common load balancing algorithms; in fact, the latter permit to split the data connection between the source and the destination by using the available connections and according to the weights assigned to each interface. In such a way, the effectiveness of the mechanism is obtained only when there are more than one end-to-end connection between the sender and the receiver. In case there is only a data flow towards the remote server, load balancing permits to assign the data stream to one of the two connections and the perceived end-to-end bandwidth is equal to the one offered by the best network operator. Instead, the use of the VPNs bond technique permits to enhance the available end-to-end bandwidth also in the case of only one data flow from the source node, i.e. the gateway of the ad hoc network, and the destination, i.e. the remote command and control position. 


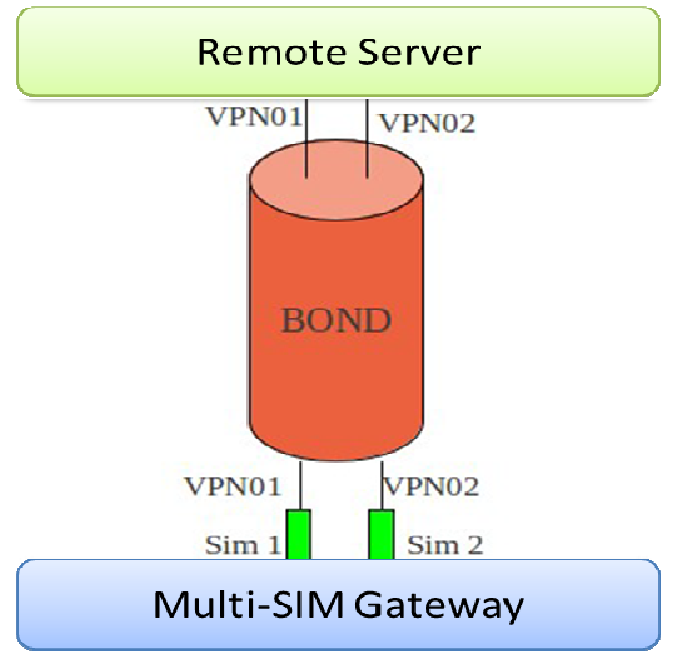

Figure 2. VPNs bond architecture.

The VPNs bonding consists in unifying two or more layer 2 connections in order to be able to assign to one data flow a bandwidth ideally equal to the sum of the bandwidth offered by single L2 connections. This technique is well known and widely employed in the field of Ethernet switches where it is possible to unify two or more L2 interfaces to guarantee a larger point-topoint bandwidth in the core network.

In the present use case, the procedure consists in the creation of a VPN between each 3G/4G interface and the end point of the communication, i.e. the remote server. Once established the two VPNs it is possible to make the bonding of the latter in order to establish a large bandwidth connection between source and destination nodes (see Figure 2). Usually the VPNs bond technique is coupled with a trivial load balancing algorithm that consists in assigning static and equal weights to each L2 connection. In case of L2 switches or DSL modem/routers this approach represents a good solution because the network conditions are similar for each interface and they remain almost constant in time. Let us suppose that the two available connections provide a bandwidth equal to $2 \mathrm{Mbps}$; in such a case a trivial load balancer will assign weights equal to 1 to each connection and the system will provide a bandwidth equal to $4 \mathrm{Mbps}$. Now, supposing that at a time $t$ the connection number 1 provides a bandwidth equal to 2 Mbps whereas the connection number 2 offers a bandwidth equal to $1 \mathrm{Mbps}$. Under this condition, if the load balancing algorithm maintains constant weights, by using a simple round robin mechanism, the connection 2 will represent a bottleneck for the system because the overall available end-to-end bandwidth will not be equal to the sum of the two bandwidths but it will be equal to twice that of the worst connection.

In a wireless scenario characterized by the high variability of radio coverage, different traffic conditions, handover procedures, and mobility, each radio interface - equipped with the related SIM - performs according to the infrastructure and the load conditions set by its operator. Under these conditions, a static weights assignment would result in a drastic reduction in performance where the worst connection would act as a bottleneck for the whole system. With the aim of overcoming this limit, the authors propose a mechanism of real time bandwidth evaluation to establish each time and for each L2 connection the weights to assign to the VPNs bond, thus maximizing the transmission rate towards the destination node.

In the next sections the adaptive load balancing algorithm will be described and the first performance evaluation of a trivial prototype will be presented. 


\section{DYNAMIC LOAD BALANCING ALGORITHM}

The proposed algorithm can be summarized in three steps:

1. Bandwidth measuremet for each available radio data connection;

2. Results analysis and weight evaluation to establish and set up the VPN bond parameters;

3. Periodic performance evaluation to determinate the most suitable weight for each mobile connection.

As to the first step, i.e. the end-to-end bandwidth evaluation, it is carried outvia the Self Loading of Periodic Streams (SloPS) technique[8][9]. The latter is based on a client-server application: the sender process runs on the multi-SIM access point whereas the receiver process runs on a remote host,which, in the present architecture, is the remote gathering node of the sensor data network. The above mentioned architecture is suitable in case the mobile hot spot has to send data towards a remote sink collecting sensor data; however, if the time-critical application is based on the transmission from the remote host to the mobile network the upload bandwith will be taken into consideration. The working principle of the Self Loading of Periodic Streams is very simple: it is based on the periodic transmission of packet streams from the source to the destination and on the consequent measure of the One Way Delay (OWD), which is the time interval between the transmission of the data packet and its reception at the receiver side. The number of packets transmitted increases until the growing one way delay; when this happens it means that the packet transmission rate is greater than the available bandwidth provided by the radio access network. Figure 3 shows the above mentioned procedure: when the transmission rate $(\mathrm{R})$ is compatible with the available bandwidth (A), i.e. $\mathrm{R}<\mathrm{A}$, the one way delay shows an almost constant trend; however, when the packet rate from the source to the destination is greater than the available bandwidth, i.e. $\mathrm{R}>\mathrm{A}$, the one way delay increases because of the TCP congestion window mechanism.
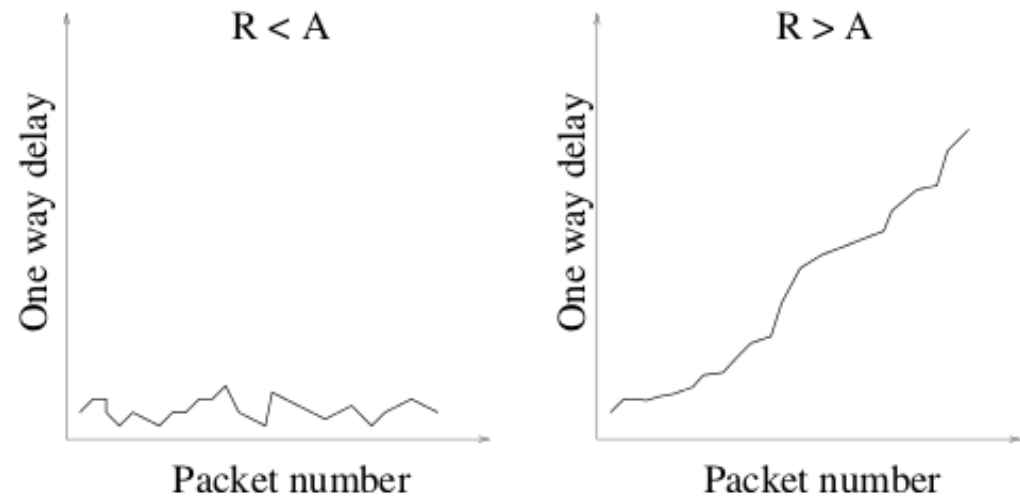

Figure 3. One way delay values of the ith packet train in the SLoPS technique[8].

The measurement is obtained by the iterative sending of a series of $K$ packets of $L$ bit each of which is transmitted during an interval of $\mathrm{T}$ seconds. In such a way the transmission rate is equal to $\mathrm{R}=\mathrm{L} / \mathrm{T}$ [bit/s]. Each packet of the series has a timestamp to indicate when the data packet has been created and sent to the receiver node. Once the destination node receives the packets stream it compares the arrival time $\left(\mathrm{A}_{\mathrm{i}}\right)$ and the sending time $\left(\mathrm{S}_{\mathrm{i}}\right)$ of the packets in order to calculate the one way delay of the ith stream, i.e. $D_{i}=A_{i}-S_{i}$. 
The sender and the receiver communicate according to the client-server paradigm in order to establish the available bandwidth. When the one way delay at the destination side increases, in fact, the receiver process notifies the sender that behaves as follows:

a. If $\mathrm{R}(\mathrm{i})<\mathrm{A}$, the source process will send the following packets stream at $\mathrm{R}(\mathrm{i}+1)>\mathrm{R}(\mathrm{i})$

b. If $\mathrm{R}(\mathrm{i})>\mathrm{A}$, the source process will send the following packets stream at $\mathrm{R}(\mathrm{i}+1)<\mathrm{R}(\mathrm{i})$

Furthermore, the rate of the stream (i+1) is established as follows [9]:

1. Two start parameters, $R_{\min }$ ed $R_{\max }$, are initialized equal to zero and equal to the ideal maximum throughput provided by the connection Rmax;

$0 \quad$ If $\mathrm{R}(\mathrm{i})<\mathrm{A}$ then $\mathrm{R}_{\min }=\mathrm{R}(\mathrm{i})$

$\circ \quad$ If $R(i)>A$ then $R_{\max }=R(i)$

2. $\mathrm{R}(\mathrm{i}+1)=\left(\mathrm{R}_{\max }-\mathrm{R}_{\min }\right) / 2$

3. The iterative process ends when $R_{\max }-R_{\min }<w$, where $w$ indicates the value depending on the precision of the bandwidth evaluation procedure (obviously, the greater the $\mathrm{w}$ the less accurate evaluation but, at the same time, the faster the convergence period required by the algorithm).

The above mentioned algorithm runs for each radio access connection and converges to the actually available end-to-end bandwidth. When the process ends, the bandwidth values are communicated to the adaptive load balancing process that updates the VPNs bond weights as follows:

a. the VPN weight of the worst connection is set equal to 1;

b. the VPN weight of the best connection is set equal to $A_{\text {better }} / A_{\text {worst }}$;

c. in case the bandwidth offered by one of the two operators is equal to 0 , e.g. because of a lack of radio coverage, the whole traffic is routed to the active connection.

The flow diagrams related to the client/server application running in the gateway side and in the remote command and control node are shown in Figure 4 and Figure 5. 
International Journal on AdHoc Networking Systems (IJANS) Vol. 5, No. 3, July 2015

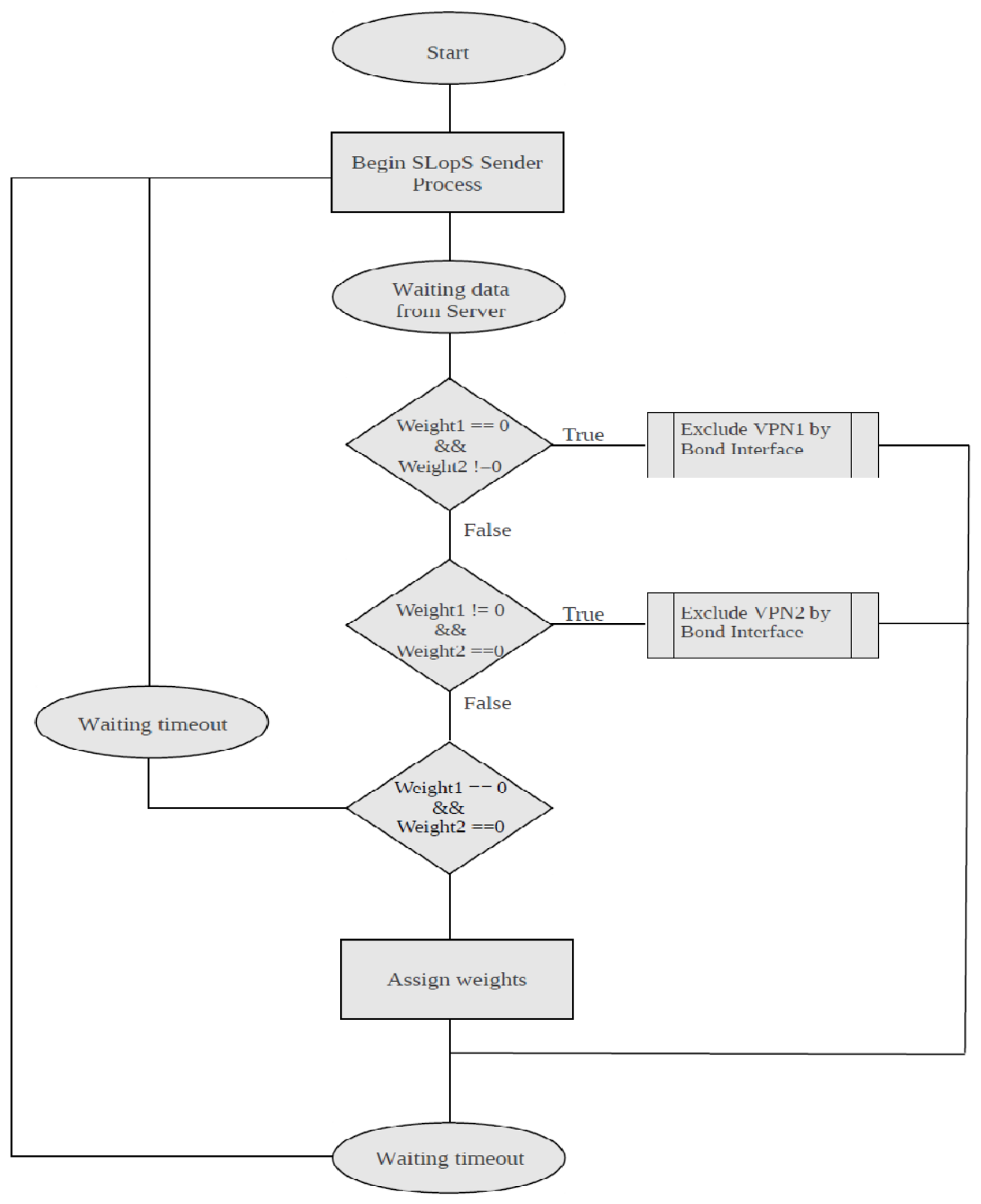

Figure 4. Bandwidth evaluation process at the mobile gateway side. 
International Journal on AdHoc Networking Systems (IJANS) Vol. 5, No. 3, July 2015

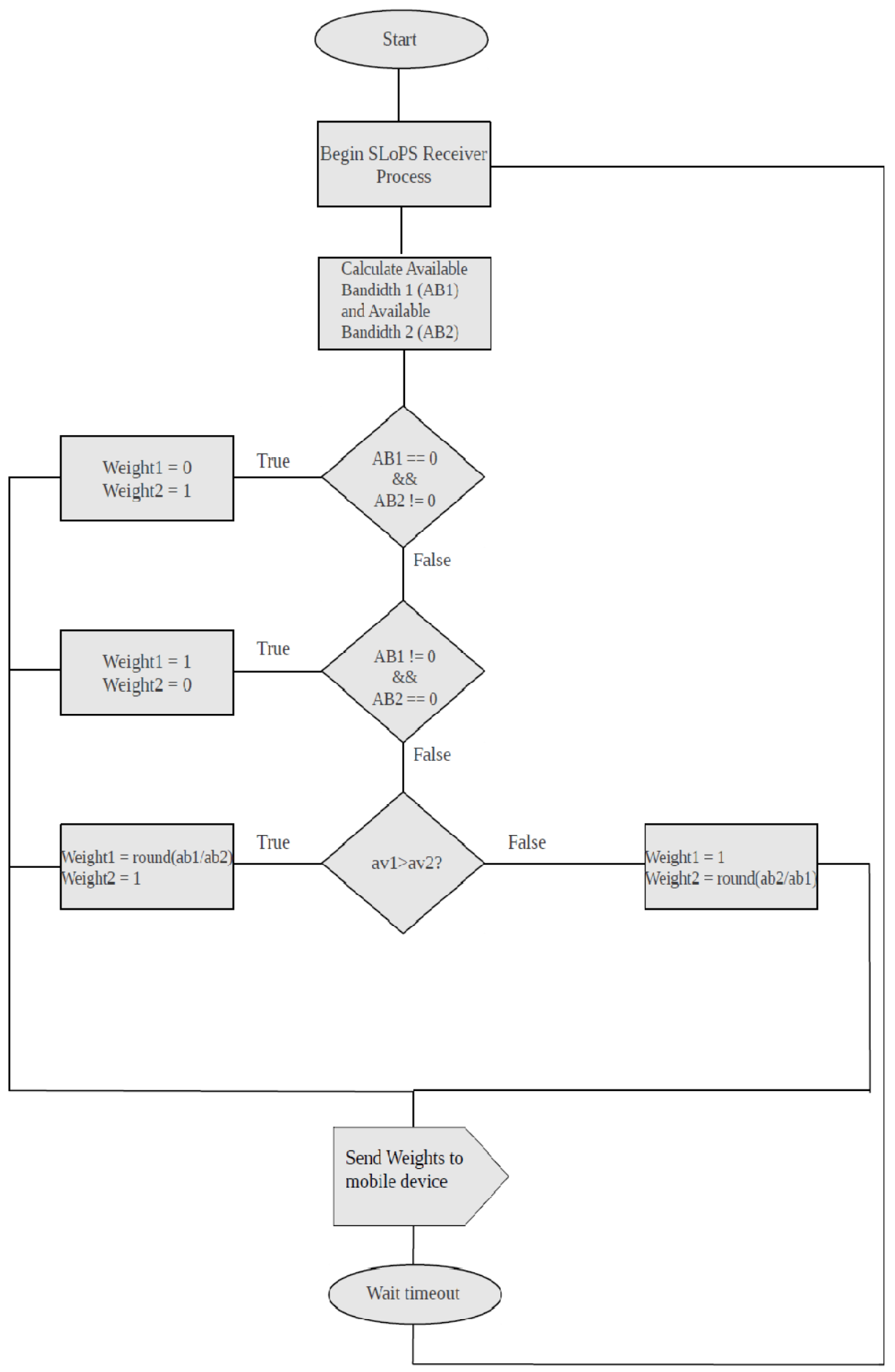

Figure5. Bandwidth evaluation process at the remote command and control side.

\section{PERFORMANCE EVALUATION}

In the above sections some problems have been illustrated that arise when a mobile wireless sensor networks have to be connected to a remote command and control server by using the 
public $3 \mathrm{G}$ or $4 \mathrm{G}$ radio network. In order to overcome such drawbacks as connection interruption, lack of bandwidth, delay in performance, jitter and packet loss rate not compatible with time critical applications, a smart dual SIM sink/gateway based on multiple radio access, VPN bonding and adaptive load balancing between the available connections is proposed. The present section will prove the effectiveness of the proposed solution showing the results obtained during the test bed. For this latter we realized a prototype by using a small form factor system boards optimized for wireless routing and network applications, an ALIX2D2 board[11], equipped with a $500 \mathrm{MHz}$ AMD Geode LX800 CPU and 256 MB DDR DRAM on board; two USB Internet keys have been used to provide cellular connectivity and the two devices have been equipped with two SIMs of two different network operators, that we will define as Operator 1 and Operator 2 in the following sections; ZeroShell[12] and OpenVPN[13] has been used as operative system and VPN manager respectively, whereas proprietary scripts has been realized by using bash and python language to evaluate the available end-to-end bandwidth between the couples source-destination and, consequently, to establish the weights to assign to each VPN in order to maximize the overall performance of the prototype. For the aim of clarity, the same hardware and software have been employed to evaluate the performance of the standard static weights assignment procedure without using, obviously, the scripts for the dynamic evaluation of the VPN weights. A picture of the hardware employed to realize our prototype is shown in Figure 6.We performed 15 data transfers from the mobile sink to the remote $\mathrm{C} 2$ evaluating the instantaneous throughput for each operator and for the one obtained by using the bond of the two VPN without the adaptive load balancing. It has been observed that the VPNs bond performs better than the two single end-toend connections providing high bandwidth connections between the mobile sensor network and the remote $\mathrm{C} 2$ position.

As shown in Figure 7, the instantaneous end-to-end throughput is not equal to the sum of the two single ones because of the presence of the overhead related to the establishment of SSH tunnel among the two radio network interfaces and the remote server.

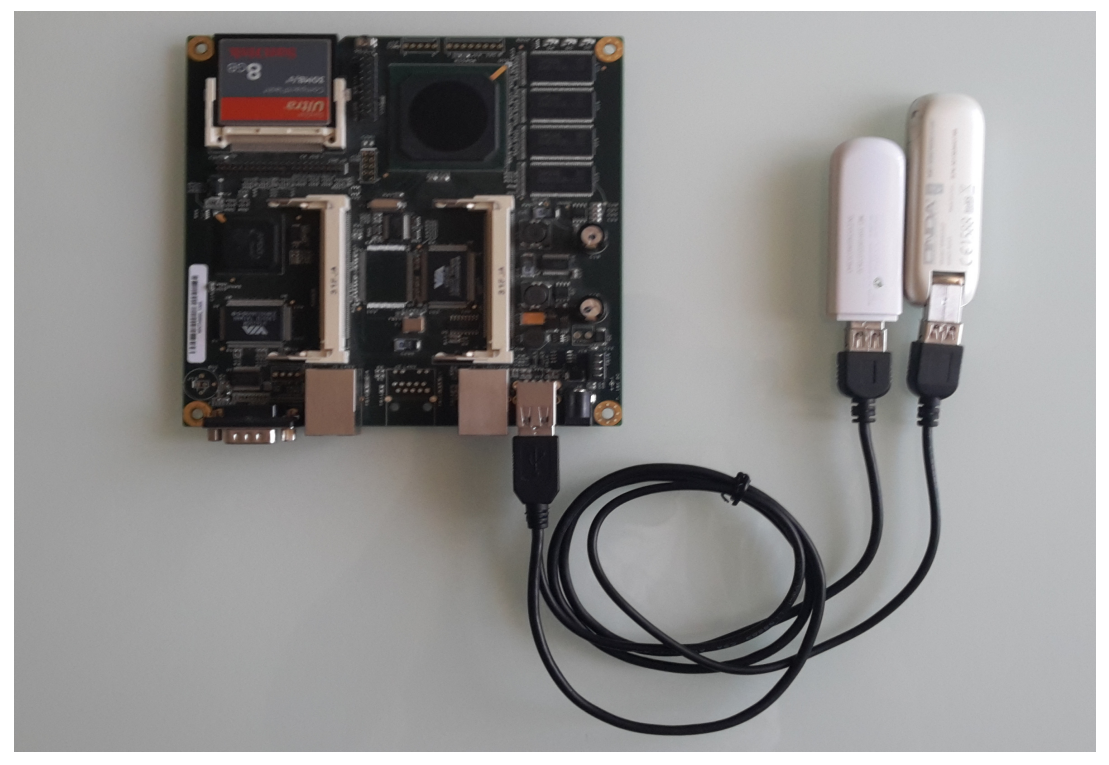

Figure 6. The prototype employed during our test bed. 
International Journal on AdHoc Networking Systems (IJANS) Vol. 5, No. 3, July 2015

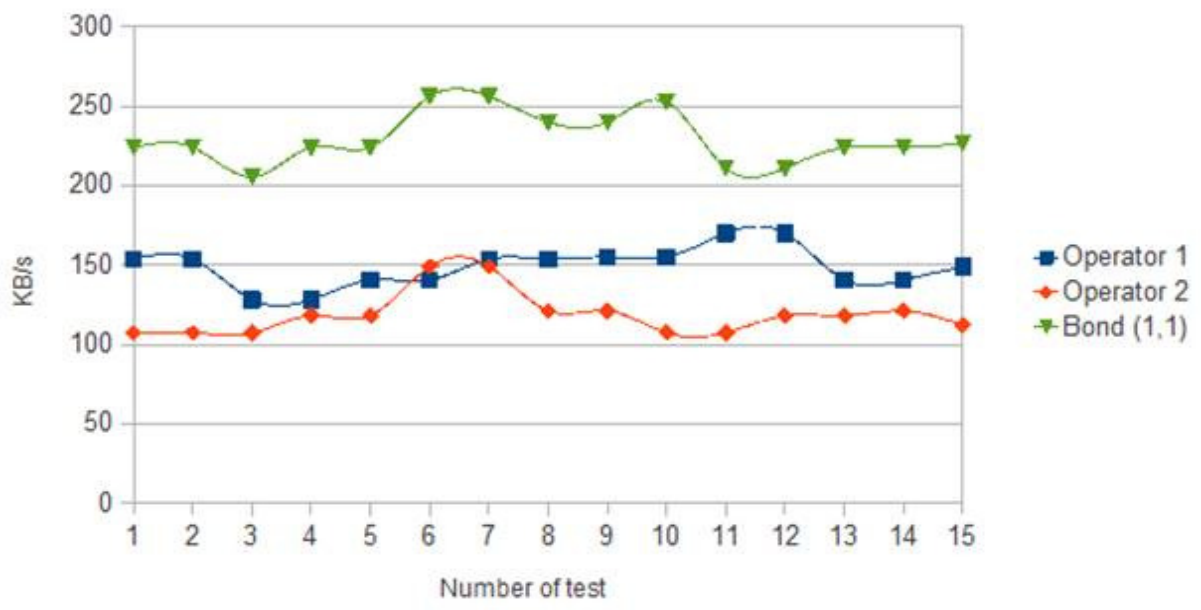

Figure 7. Available bandwidth perceived by a single TCP connection.

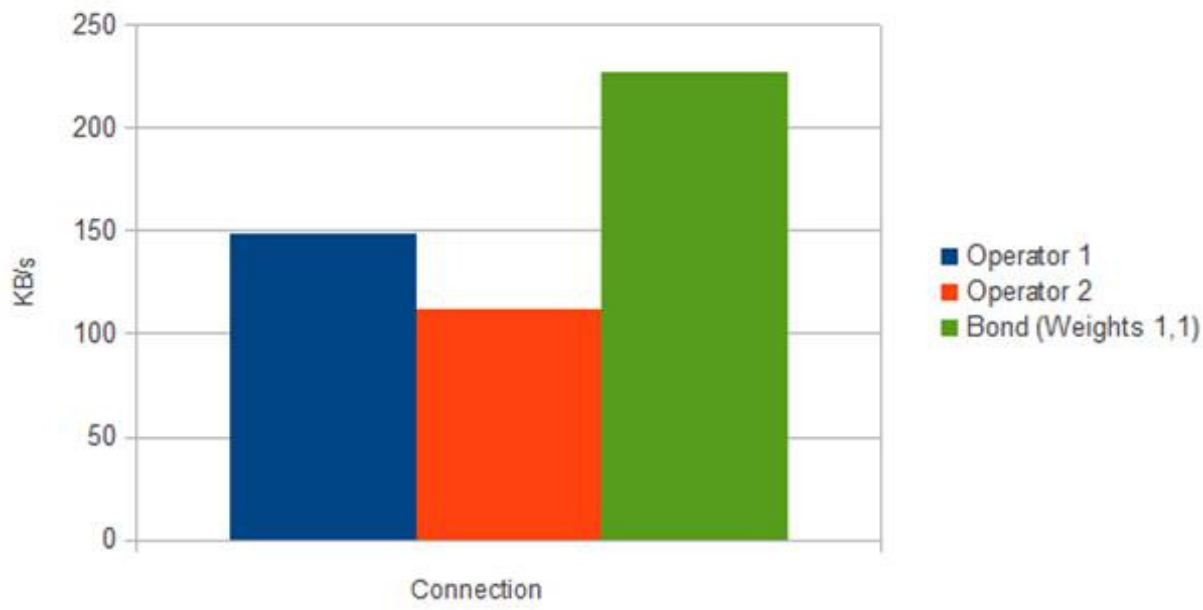

Figure8. Average end-to-end bandwidth comparison.

To summarize (refer to Figure 8), the use of a dual SIM sink/gateway permits to enhance the available end-to-end bandwidth by more than $50 \%$ if compared to the best radio operator, Operator 1, and more than $100 \%$ if compared to the worst radio operator in our test bed Operator 2 .

Obviously, the use of two or more network accesses guarantees a seamless connectivity between the source and the destination if compared to the use of only one radio interface. Figure 9 shows the instantaneous bandwidth of the two network connections and the VPN bond; as it appears when one of the two operators goes down due to network congestion or lack of radio coverage the VPN bond performs as the only working operator and the prototype behaves every time like a common single stream device equipped with the USIM belonging to the best network operator, i.e. the operator offering the best connectivity at the given time.

The performance delivered by the VPN bond coupled with the static load balancing between the two available connections is satisfactory in the above mentioned scenarios; however, this technique has some drawbacks when one of the two operators does not go down but, more simply, offers an end-to-end bandwidth variety during the sink movement due to such reasons as 
radio coverage, traffic congestion, handover procedures and so on. It can appear that during the sink movement the network operator 2 delivers almost constant performance whereas the network operator 1 shows a drastic reduction in the bandwidth see Figure 10). In such a case, the VPN bond and the static load balancing algorithm does not perform as expected and the dual SIM gateway behaves as the worst of the two network operators; in fact, the load balancing algorithm splits into two equal flows, the original one assigning to each connection the amount of data to transmit equal to the available bandwidth offered by the worst operator.

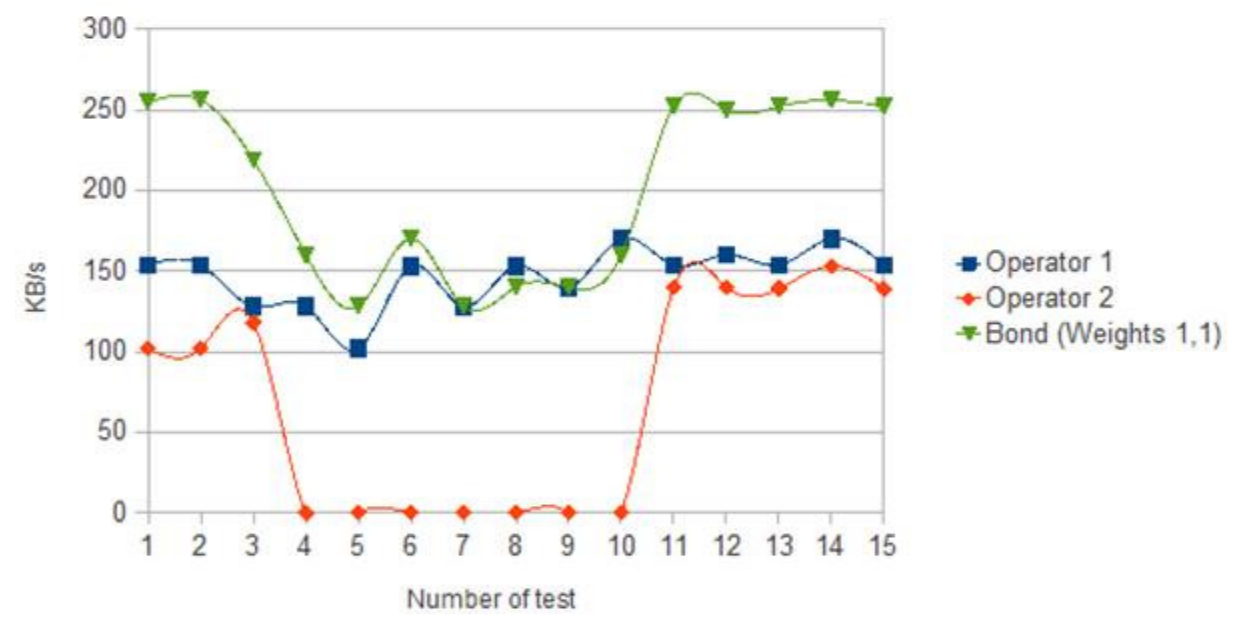

Figure 9. Available bandwidth perceived by a single TCP connection during Operator 2 failure.

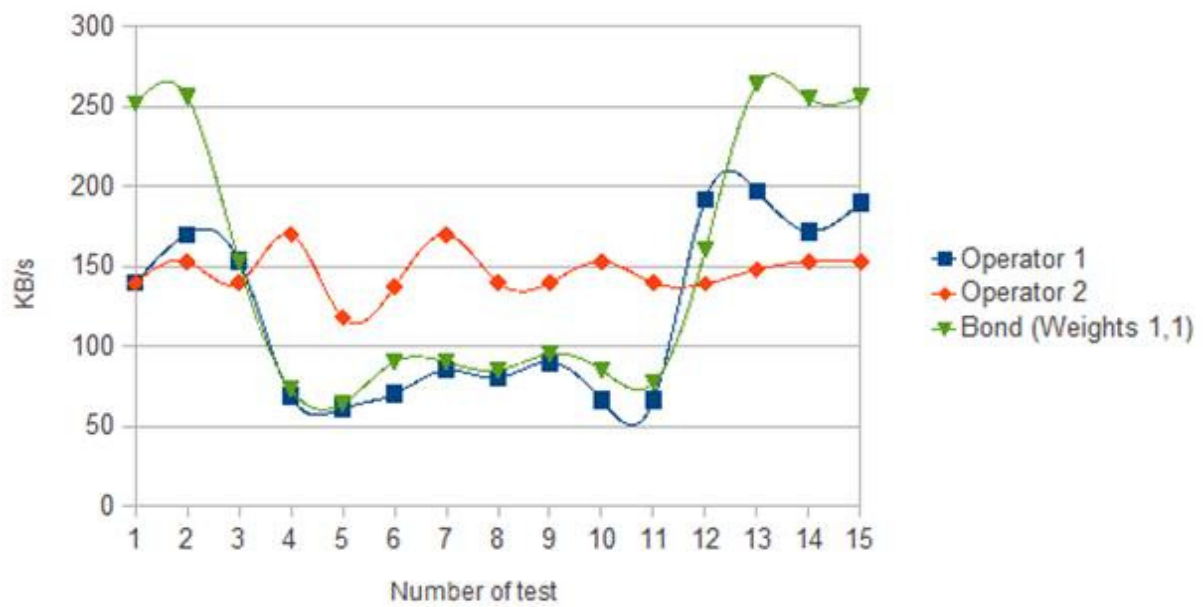

Figure10. Available bandwidth perceived by a single TCP connection during Operator 1 bandwidth degradation in case of static weights.

Figure 10 shows the performance delivered by using the connections offered by Operator 1, Operator 2 and the traditional VPN bond technique respectively. As shown in the figure, the dual SIM prototype behaviour is similar to that of the worst operator; the latter representing, in fact, the bottleneck of the system. In such a case, the VPN bond coupled with the load balancing does not offer any performance enhancement because of the incorrect assignment of bond weights.

Figure 11, instead, indicates the results obtained by the proposed cellular bonding prototype with the use of an adaptive load balancing algorithm in order to counteract the drawbacks related to the variability of the end-to-end bandwidth offered by each radio operator during the movement of the mobile sink/gateway. The mobile sink/gateway was moved following the same route. It appears that the cellular bonding almost always outperforms the best cellular operator; however, 
International Journal on AdHoc Networking Systems (IJANS) Vol. 5, No. 3, July 2015

when this does not happen it is due to the convergence time of the adaptive weights algorithms. Also,this case highlights that the final end-to-end bandwidth is not equal to the sum of all bandwidths because of the presence of the overhead due to the implementation of the VPNs and their bonding. In the Figure 12 the behaviour of static and dynamic weights is presented, whereas in the Figure 13 the average end-to-end bandwidth of the two schemes is compared. As we can see, the VPN bond with adaptive weights assignment outperforms the static assignment scheme by almost $100 \%$ showing the effectiveness of the proposed solution in vehicular applications such as telemedicine, telemetry, remote command and control, etc.

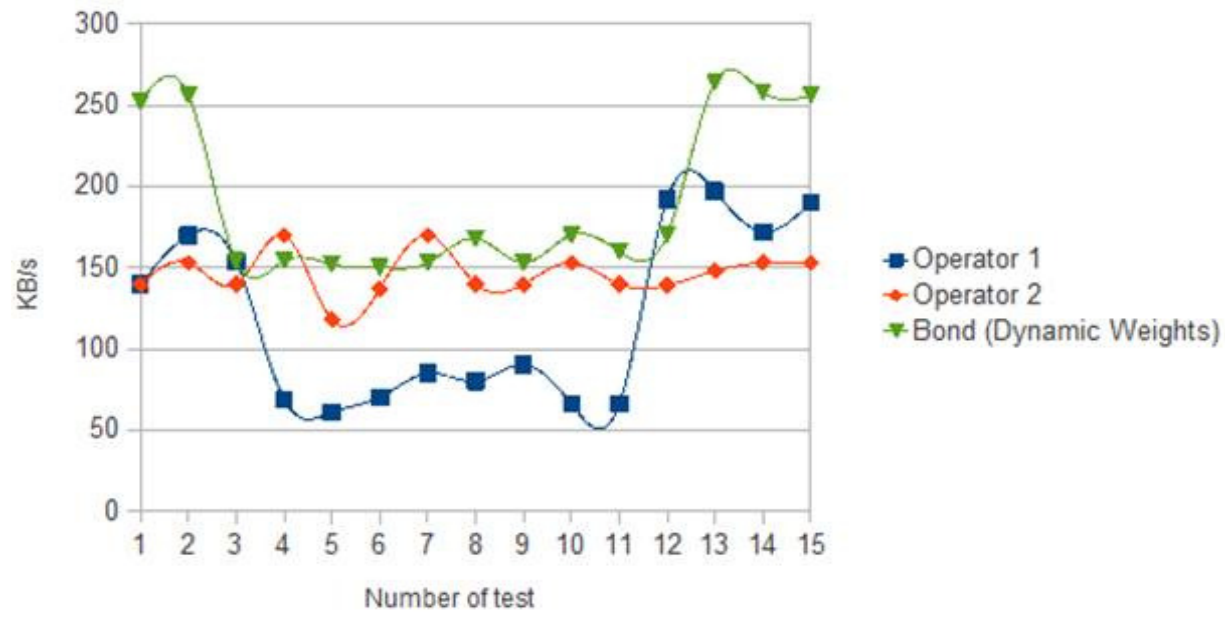

Figure 11. Available bandwidth perceived by a single TCP connection during Operator 1 bandwidth degradation in case of adaptive weights.

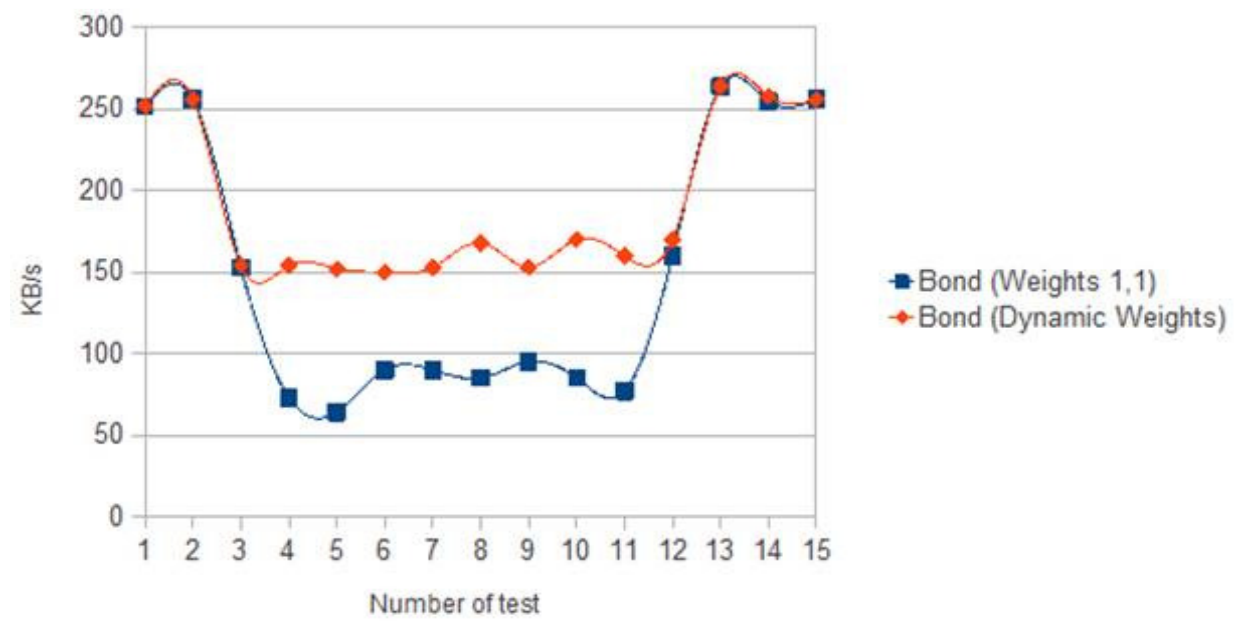

Figure12. Bandwidth comparison between VPNs bond with static and dynamic weights. 


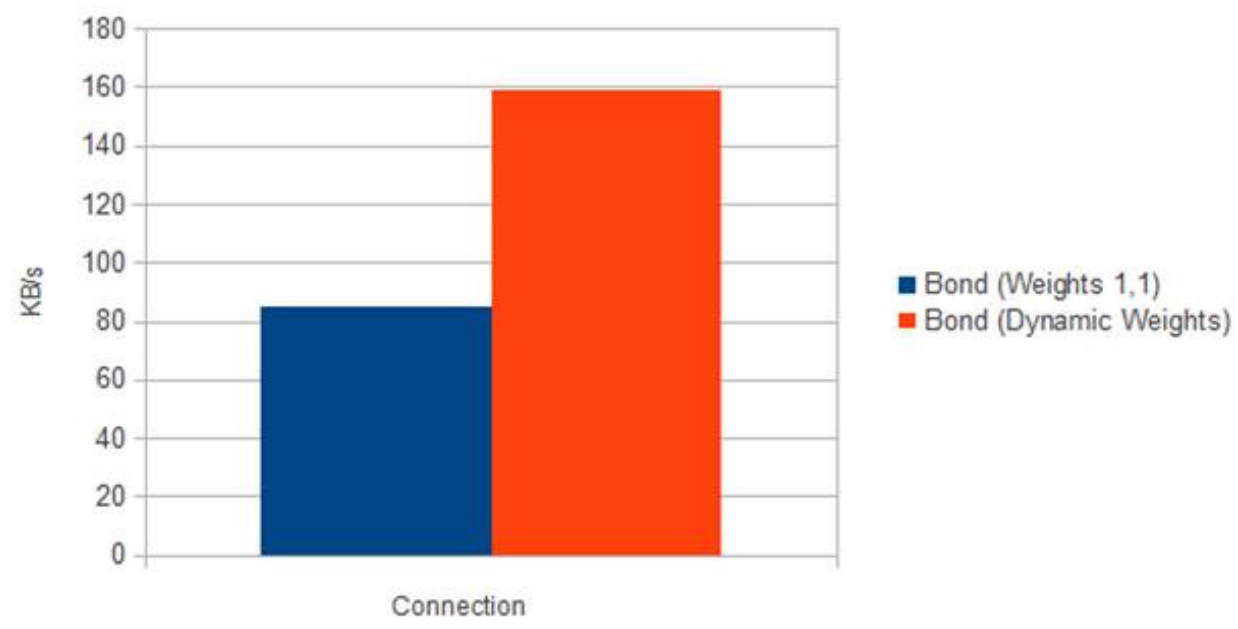

Figure13. Average bandwidth comparison between VPNs bond with static and dynamic weights.

\section{CONCLUSION}

The present paper proposes a smart gateway for mobile wireless sensor networks, IoT networks or local area networks for time critical or real time communications based on two approaches, the VPNs bonding and the dynamic load balancing between the available radio access connections. The first approach permits to enhance the available end-to-end bandwidth and the reliability of the connection between the sink and the remote position; the second step consists in the dynamic weights calculation to be assigned to each connection in order to maximize the cumulative endto-end bandwidth. In fact, Mobile IP networks are not designed to support real-time and/or timecritical traffic because of several drawbacks concerning the wireless medium, such as resources sharing, traffic congestion, radio link coverage etc., which impact directly such parameters as bandwidth, delay, jitter, and packet losses that are the main causes of quality degradation of numerous services over the PSTN. Under this condition, the dynamic assignment of the weight to each connection of the VPN bonding plays a key role in exploiting the best connection available at the given time. Performance evaluation of the prototype shows the effectiveness of our approach in terms of instantaneous throughput. Considering the future work, the authors of the present paper are currently working on a device that is able to calculate the dynamic weights of the load balancing algorithm based on the kind of data traffic the sink/gateway has to transmit or receive from the remote command and control station.

\section{ACKNOWLEDGEMENTS}

This work has been partially supported by the "ProgrammaOperativoNazionale "Ricerca\&Competitività" 2007-2013" within the project "PON04a2_E - SINERGREEN - RES NOVAE - Smart Energy Master per ilgovernoenergetico del territorio" and the project "PON01_00683 - SIGMA - Sistema Integrato di sensori in ambiente cloud perlaGestioneMultirischioAvanzata". 
International Journal on AdHoc Networking Systems (IJANS) Vol. 5, No. 3, July 2015

\section{REFERENCES}

[1] A. Lamba, J. Yadav, G. U. Devi, "Analysis of Technologies in 3G and 3.5G Mobile Networks", in Proc. of Int. Conf. on Communication Systems and Network Technologies (CSNT), 11-13 May, 2012, pp. 330-333.

[2] F. Beritelli, A. Gallotta, C. Rametta, “A Dual Streaming Approach for Speech Quality Enhancement of VoIP service over 3G networks", Proceedings of IEEE Digital Signal Processing (DSP), 1-3 July 2013.

[3] F. Beritelli, A. Gallotta, S. Palazzo, C. Rametta, "Dual stream transmission to improve mobile VoIP services over HSPA: a practical test bed", Proceedings of IEEE 8th International Symposium on Image and Signal Processing and Analysis (ISPA), 4-6 September 2013.

[4] F. Beritelli, C. Rametta, "HSDPA Dual Streaming Approach for Improving VoIP Speech Quality in Forensic Applications", Proceedings of IEEE 9th International Symposium on on Communications Systems Networks and Digital Signal Processing (CSNDSP) 2014, 23-25 July 2014, Manchester, UK.

[5] F. Beritelli, C. Rametta, "A Mobile Dual VoIP System for Enhancing Speech Quality and Intelligibility: Simulation and Test Bed”, Transactions on Networks and Communicat ions; Volume 3, Issue 2, April 2015.

[6] J. Yick, B. Mukherjee, D. Ghosal, "Wireless sensor network survey", Elsevier Computer Networks, Volume 52, Issue 12, 2008.

[7] L. Atzori, A. Iera and G. Morabito, "The Internet of Things: A survey”, Elsevier Computer Networks, Volume 54, Issue 15, 2010.

[8] Manish Jain, Constantinos Dovrolis, "Pathload: a measurement tool for end-to-end available bandwidth",in Proc. of Passive and Active Measurements (PAM) Workshop, 2002

[9] R.S. Prasad, M.Murray, C.Dovrolis, K.Claffy, "Bandwith Estimation: metrics, measurement techniques and tools", IEEE Network, Nov 2003.

[10] Manish Jain, Constantinos Dovrolis, "End-to-end Available Bandwith: Measurements, Methodology, Dynamic and Relation with TCP Throughput”, IEEE/ACM Transactions on Networking, Ago 2003.

[11] http://www.pcengines.ch/alix.htm

[12] http://www.zeroshell.net

[13] Openvpn: Building and Integrating Virtual Private Networks, Markus Feilner, Packt Publishing Ltd, 11 mag 2006.

\section{Authors}

Francesco Beritelli received the Laurea degree in Electronic Engineering and the Ph.D. degree in Electronics, Computer Science, and Telecommunications Engineering from the University of Catania, Catania, Italy, in 1993 and in 1997, respectively. From 1997 to 2000 in collaboration with CSELT (now Telecom Italia LAB) he took an active part in international ITU-T standardization meetings. From 1998 he is the founding member of the "Multimedia Technologies Institute - MTI", applied research laboratory operating in DSP (Digital Signal Processor) and CTI (Computer Telephony Integration) applications.

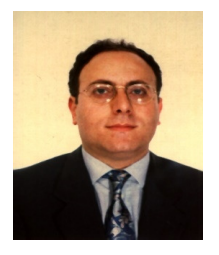
Currently, from 2002, he is Assistant Professor in the Department of Electric, Electronic and Computer Science Engineering at the University of Catania. His main research activities are in the area of robust audio and speech signal classification and recognition, variable bit-rate speech coding, and adaptive-rate voice and dual stream transmission for mobile IP telephony applications. His interests also include the field of biometric identification and cardiac signal processing. He is a member of the Institute of Electrical and Electronic Engineers (IEEE) and the "IEEE Signal Processing Society" since 1996 and has 100 scientific publications, mainly in international journals, books and conference proceedings.

Aurelio La Cortereceived the M.Sc. degree in electrical engineering from the University of Catania in 1988, and the Ph.D. in Electronic Engineering and Computer Science in 1994. From 1994 he is at the University of Catania. He is an associate professor in Telecommunication Engineering. His scientific interests include network and QoS management techniques, risk analysis of ICT systems, bio-inspired models for information security, protocols and architecture for integrated communications.

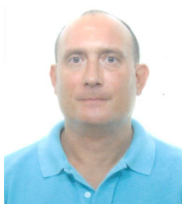


International Journal on AdHoc Networking Systems (IJANS) Vol. 5, No. 3, July 2015

CorradoRametta received the Laurea degree in Electronic Engineering and the Ph.D. degree in Computer Science and Telecommunications Engineering from the University of Catania, Italy, in 2008 and in 2012, respectively. From 2009 to 2012, he was a Research Engineer in the field of wireless communications with the CNIT (ConsorzioNazionaleInteruniversitario per le Telecomunicazioni). From 2012 he works as a post-doc researcher at the University of Catania. His research interests include wireless

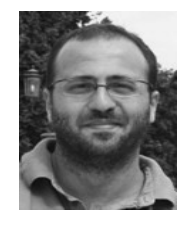
mesh networks, ad-hoc and sensor networks, modeling and simulation of communications protocols, software defined networking, network function virtualization and embedded systems for multimedia applications.

Francesco Scaglionereceived the Laurea degree in Telecommunications Engineering from the University of Catania in 2011. His research interests include embedded systems, VoIP communications and ICT technologies for environmental control and monitoring.

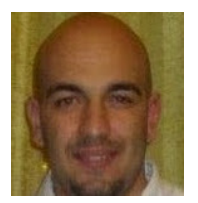

